presence of an activator domain which promotes the step. This suggests that the addition of a component that binds to promoter DNA in a nonspecific fashion (that is, chromatin proteins) could under some conditions satisfy some of the assay properties suggested previously for coactivators. In a mechanistic sense, little is known about the kinetics of TFIIB binding to the TFIIDtemplate complex. The specific footprint generated by the binding of TFIIB over the initiation site is not pronounced and is confined to one strand of the template ${ }^{4}$, so TFIIB may not be strongly bound to the template in the absence of an activator domain.

Final answers to most of the questions addressed by these experiments await the purification and analysis of the specific proteins that constitute TFIIB and the other basal components. The most purified preparations of TFIIB contain a protein of relative molecular mass 32,000 , and specific antiserum has been prepared ${ }^{11}$ which reacts with pro- tein in the template TFIID-TFIIB complex. It is highly likely that a complementary DNA encoding this factor will be available shortly, and more refined studies of the interaction of the protein with the acidic activator and TFIID will then be possible.

Phillip A. Sharp is in the Center for Cancer Research and the Department of Biology, Massachusetts Institute of Technology, Cambridge, Massachusetts 02139, USA.

1. Workman, J. L., Taylor, I. C. A. \& Kingston, R. E. Cell 64 533-544 (1991).

2. Croston, G. E., Kerrigan, L. A., Lira, L. M., Marshak, D. R. \& Kadoriaga, J. T. Science 251, 643-649 (1991).

3. Lin, Y.-S. \& Green, M. R. Cell 64, 971-981 (1991).

4. Buratowski, S., Hahn, S., Guarente, L. \& Sharp, P. A. Cell 56, 549-561 (1989)

5. Hope, I. A. \& Struhl, K. Cell 46, 885-894 (1986).

6. Ptashne, M. Nature 335, 683-689 (1988).

7. Cress, W. D. \& Triezenberg, S. J. Science 251, 87-90 (1991).

8. Lewis, B. Cell 61, 1161-1164 (1990).

9. Schlissel, M. S. \& Brown, D. D. Cell 37, 903-912 (1984) 0. Stringer, K. F., Ingles, C. J. \& Greenblatt, J. Nature $\mathbf{3 4 5}$, 783-786 (1990)

11. Maldonado, E., Ha, I., Corest, P., Weis, L. \& Reinberg, D. Molec. cell. Biol. 10, 6335-6347 (1990).

\title{
A new act to swallow
}

\section{Eve Marder}

ON page 60 of this issue ${ }^{1}$, Meyrand and colleagues demonstrate that the same neurons can be part of several different functional networks, some of which may only exist operationally for a short time if the behaviour they control is not continuous. This contrasts with the view that each neuron has a specific and unique role in sensory processing or motor function; for example, one might imagine that the networks that generate walking are activated during walking, but when the animal is not walking these neurons are not used. The view of the organization of the nervous system that Meyrand et al. now provide shows surprising similarity to one seen in neural network theory, in which individual elements can be used to construct many different functional networks.

Meyrand et al. studied the neural networks of the crustacean stomatogastric nervous system $^{2}$, which consists of four ganglia: the stomatogastric ganglion with 30 neurons, the oesophageal ganglion of 18 neurons, and the two commissural ganglia, each with several hundred neurons. When removed from the animal, the stomatogastric system retains the ability to generate several different rhythmic motor patterns. These include the pyloric rhythm (0.5-2-second period), the gastric

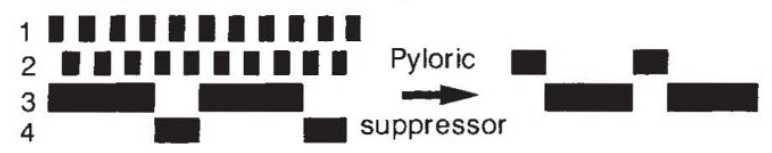

Block diagrams showing the activity of different neural elements. Traces 1 and 2, pyloric elements; traces 3 and 4 gastric elements. Under control conditions (left) the faste pyloric and slower gastric rhythms occur simultaneously. Pyloric suppressor activity evokes a novel pattern using elements of both the fast and slow rhythms. Other ele ments become inactive (traces 1 and 4). and oesophageal rhythms (each of 5-10second period), the cardiac-sac rhythm (30-120-second period), and a swallowing pattern described for the first time in the new work ${ }^{1}$. The cellular properties and circuit interactions responsible for generating the pyloric and gastric rhythms are relatively well understood because the neurons involved are relatively few in number, and are large and uniquely identifiable regardless of their firing patterns, and because the motor neurons themselves are important components of the pattern-generating networks.

For many years biologists have treated the stomatogastric nervous system as if it were divided into neat, almost independent, subsystems, each responsible for the generation of a different motor pattern. In this view, neurons participate in only one motor pattern, and are classified as 'pyloric' or 'gastric' on the basis of their anatomical projections and firing patterns. But then Hooper and Moulins ${ }^{3}$ showed that the ventricular dilator neuron could switch its firing pattern between the pyloric and the cardiac-sac rhythm. And Weimann et al. ${ }^{4}$ demonstrated that many neurons previously thought to participate exclusively in the generation of the pyloric or gastric rhythms could fire in time with either rhythm. Furthermore, Dickinson et al. ${ }^{5}$ showed that a peptide (red pigment concentrating hormone) could elicit a novel rhythm in which gastric and cardiac-sac neurons were coordinately active. Meyrand et al. now show that, when the animal swallows, a pair of py- loric suppressor neurons are activated which override the synaptic and cellular events responsible for the generation of the pyloric, gastric and oesophageal rhythms, and which recruit some of the elements of these networks to make a new network to generate the swallowing motor patterns.

The pyloric suppressor neurons can be activated by stimulating sensory pathways. The neurons then act at many sites in the stomatogastric nervous system: they drive motor neurons that control the opening of the oesophageal valve for swallowing; strongly inhibit many of the neurons of the pyloric and gastric networks; and strongly excite other neurons of the pyloric, gastric and oesophageal networks. The net result is that bursts of action potentials in the pyloric suppressor neurons evoke a new motor pattern in which neurons of the pyloric, gastric and oesophageal networks fire coordinately with each other and with the neurons that operate the oesophageal valve (see figure).

It is important to remember that the stomatogastric nervous system is different from many in that the motor neurons themselves are intrinsic components of the central pattern-generating circuits. Therefore, when an identified stomatogastric ganglion motor neuron alters its activity pattern, the behaviourally relevant output and the effect of neurons that shape this output are seen simultaneously. So the new studies show that the same neuron can participate in several distinct neural networks. They also demonstrate that operational networks can be formed by synaptic and modulatory inputs when needed, but that their elements can be freed for other tasks at other times.

Some may be tempted to argue that the kinds of circuit reconfigurations that can be seen in invertebrates are not necessary in the vertebrate nervous system, with its vastly larger number of neurons. I remember when a similar argument was made about the ability of invertebrate neurons to generate bursting pacemaker and plateau potentials. Now we know that vertebrate neurons can display as rich a range of membrane properties as has been long known for invertebrate neurons. I await the time when workers on vertebrate preparations can reliably identify neurons independently of their firing patterns. We will then see the extent to which neurons in the vertebrate nervous system are used as elements in many different functional circuits.

Eve Marder is in the Department of Biology and Center for Complex Systems, Brandeis University, Waltham, Massachusetts 02254, USA.

1. Meyrand, P., Simmers, J. \& Moulins, M. Nature 351, 60-63 1991).

2. Selverston, A, I. \& Moulins, M. (eds) The Crustacean Stomatogastric Nervous System (Springer, Berlin, 1987).

3. Hooper, S. L. \& Moulins, M. Science 244, 1587-1589 (1989)

4. Weimann, J. M., Meyrand, P. \& Marder, E. J. Neurophysiol. 65, 111-122 (1991).

5. Dickinson, P. S., Mecsas, C. \& Marder, E. Nature 344 155-158 (1990). 